Research Paper

\title{
Differential diagnostic roles of the serum CA19-9, total bilirubin (TBIL) and the ratio of CA19-9 to TBIL for benign and malignant
}

\author{
Wei Liu*, Qiaofei Liu*, Wenqin Wang*, Penghui Wang, Jieming Chen, Tao Hong, Ning Zhang, Binglu Li, \\ Qiang Qu, Xiaodong $\mathrm{He}^{\bowtie}$ \\ Department of General Surgery, Peking Union Medical College Hospital, Chinese Academy of Medical Sciences \& Peking Union Medical College,100730, \\ Beijing, China. \\ *These authors contributed equally. \\ $\bowtie$ Corresponding author: Prof. He XD, E-mail: hexdpumch@sina.com \\ (c) Ivyspring International Publisher. This is an open access article distributed under the terms of the Creative Commons Attribution (CC BY-NC) license \\ (https://creativecommons.org/licenses/by-nc/4.0/). See http://ivyspring.com/terms for full terms and conditions.
}

Received: 2018.01.22; Accepted: 2018.03.08; Published: 2018.04.19

\begin{abstract}
Background: Obstructive jaundice is one of the most common symptoms which can be caused by both malignant and benign hepato-biliary-pancreatic diseases. The differences and the differential diagnostic roles of the serum CA19-9, total bilirubin (TBIL) and especially, the ratio of CA19-9 to TBIL in these patients have not been well elucidated. This study compared the differences and the differential diagnostic roles of the increase-folds of the serum CA19-9, TBIL and the ratio of increase-folds of CA19-9 to increase-folds of TBIL in 508 cases of malignant (MOJ) and benign (BOJ) obstructive jaundice patients.

Methods: Totally, 508 cases of obstructive jaundice patients with pathological results were retrospectively enrolled. 342 cases were MOJ, including gallbladder adenocarcinoma, extrahepatic cholangiocarcinoma, periampullar adenocarcinoma and pancreatic adenocarcinoma.The other 166 cases were BOJ, including cholelithiasis, primary sclerosing cholangitis (PSC), IgG4 related sclerosing cholangitis (IRSD), inflammatory stricture of bile duct, and adenoma. The data of the increase-folds of serum CA19-9, TBIL and the ratio of increase-folds of CA19-9 to increase-folds of TBIL was collected and analyzed. The ROC (receiver operating characteristic) curve was adopted to determine the optimal cutoff value to evaluate their differential diagnostic roles.

Results: The CA19-9 was elevated in $94.15 \%$ of the MOJ, compared to $67.10 \%$ in $\mathrm{BOJ}(P<0.0001)$. The increase-folds of the CA19-9 $(76.52 \pm 15.04$ vs $3.55 \pm 0.41, P<0.0001)$ and the increase-folds of TBIL $(7.10 \pm 0.25$ vs $5.95 \pm 0.36, P=0.0034)$ and the ratio $(14.96 \pm 5.05$ vs $0.79 \pm 0.93, P=0.0026)$ in $M O J$ were significantly higher than BOJ. The ROC curves indicated that the TBIL showed minimal differential diagnostic power (AUC $=0.590)$, the increase-folds of CA19-9 and the ratio showed stronger differential diagnostic power (AUC=0.815 and $A \cup C=0.889$, respectively). The optimal value of the increase-folds of serum CA19-9 and the ratio for differential diagnosis was 4.5 and 0.88 , respectively. Further, the combination of the increase-folds of serum CA19-9 and the ratio could increase the specificity and accuracy of the diagnosis of MOJ.

Conclusion: The serum CA19-9, TBIL and the ratio in MOJ and BOJ are significantly different. The ratio of the increase-folds of CA19-9 to increase-folds of TBIL has stronger differential diagnostic roles than the increase-folds of CA19-9 or TBIL alone. The combination of the increase-folds of the CA19-9 and the ratio can further improve the specificity and accuracy of the diagnosis of $\mathrm{MO}$.
\end{abstract}

Key words: Obstructive jaundice; Bilirubin; CA19-9; Differential diagnosis; pancreatic cancer; IgG4 related sclerosing cholangitis

\section{Introduction}

Obstructive jaundice is one of most common symptoms which can be caused by both benign and malignant hepato- biliary - pancreatic diseases. Accurate differential diagnosis of obstructive jaundice 
is of great clinical importance [1, 2]. The CA19-9 (carbohydrate antigen 19-9) is a carbohydrate tumor-associated antigen which is frequently up regulated in malignant obstructive jaundice (MOJ), however the elevation of CA19-9 in malignancies can be disturbed by obstructive jaundice and biliary inflammation[3, 4]. Due to the mechanical obstruction of the biliary duct, the CA19-9 in the bile which is secreted by normal biliary or pancreatic ductal epithelium will reverse into blood and the secondary inflammations may also stimulate normal biliary or pancreatic ductal epithelium to secrete more CA19-9, which further contributes to the elevation of serum CA19-9. Malignant cancer cells can uncontrollably secrete CA19-9 which could be the majority portion of the elevation of serum CA19-9. Under most circumstance, the serum CA19-9 in benign obstructive jaundice (BOJ) is less than $100 \mathrm{U} / \mathrm{ml}$, however, the serum CA19-9 in some of the cases can be more than $500 \mathrm{U} / \mathrm{ml}$, or even more than $1000 \mathrm{U} / \mathrm{ml}$, therefore, the CA19-9 elevation alone is insufficient for differential diagnosis of $\mathrm{MOJ}$ and BOJ[5, 6]. Several strategies have been recommended to improve the power of differential diagnostic roles of CA19-9, including a higher cutoff value, combination with some other markers and adjustment of CA19-9 by other markers. La Greca et al [3]reported that when the CA19-9 value was adjusted by dividing by $C$ reactive protein(CRP), the diagnostic power of the CA19-9 was improved in 102 cases of obstructive jaundice patients. Kaur et al [7] reported the combination of mucin 5AC and CA19-9 significantly improved the diagnostic accuracy for differentiating resectable pancreatic cancer from the controls. However, these previous studies had a limited number of patients and an incomplete spectrum of etiologies of $\mathrm{MOJ}$ and $\mathrm{BOJ}$. The elevation of CA19-9 in the BOJ, the trigger of elevated CA19-9 was the mechanical obstruction of biliary tract, however, in the MOJ, the mechanical obstruction of biliary tract and cancer cell-secreted CA19-9 contributed together to the elevation of serum CA19-9. Theoretically, the ratio of increase-folds of CA19-9 to the increase-folds of TBIL, which could minimize the disturbance of mechanical obstruction, should have a stronger power for differential diagnosis of MOJ, however, this assumption has never been validated.

IgG4 related sclerosing cholangitis (IRSC), a rare newly defined autoimmune disease of bile duct in 2009, which could lead to obstructive jaundice, has got more and more attentions[1]. IRSC responses well to the steroids treatment and should not be treated by operation. Sometimes, differential diagnosis of IRSC and MOJ can be very difficult $[8,9]$. The differential diagnostic roles of the serum CA19-9 has not been reported between IRSC and MOJ. In this study, we enrolled 508 cases of obstructive jaundice patients, including 78 cases of IRSC, which could be the largest single center report. In this study, $508 \mathrm{MOJ}$ and BOJ patients with a complete spectrum of etiologies, especially including 76 cases of IRSD, were enrolled. The differences and the differential roles of the elevation of CA19-9, the elevation of TBIL and the ratio of the elevation of CA19-9 to the elevation of TBIL were systematically analyzed. The assumption that the ratio can improve the power of the elevation of CA19-9 or TBIL alone to discriminate between MOJ and BOJ was initially validated.

\section{Patients and methods}

\section{Patients and diagnosis}

All of the patients were inpatients and were admitted for obstructive jaundice to Peking Union Medical College Hospital. The clinical-pathological information of the patients was extracted from the PACS system. The upper threshold of normal serum TBIL was $22.2 \mu \mathrm{mol} / 1$ and the upper threshold of normal serum CA19-9 was 34U/L. Obstructive jaundice was defined as the serum TBIL was over the upper threshold of normal range and any imaging modality showed signs of obstruction of biliary tract. The value of CA19-9 and TBIL was collected before bile drainage and the ratio of CA19-9/34U/L to TBIL/22.2 $\mu \mathrm{mol} / 1$ was calculated. All of the MOJ patients were pathologically confirmed to be adenocarcinoma, by surgical resection or biopsy. Among the 166 cases of BOJ, different diagnostic modalities were adopted. All of the cholelithiasis patients underwent endoscopic retrograde cholangiopancreatography or intraoperative choledochoscope and the biopsy was performed when malignancy was suspected. All of the adenomas located in periampullar or bile duct were surgically en bloc resected and the final pathological results showed no adenocarcinoma. All of the inflammatory strictures of bile duct were pathologically confirmed. Primary scleoring cholangitis (PSC) was diagnosed according to the guideline of American Association for the Study of Liver Diseases or the consensus of the Chinese Association for Liver Disease and Chinese Association for Digestive Disease[10, 11]. IRSC was diagnosed according to the consensus of Japanese Biliary Association[12] and all of the cases reached the criteria of probable diagnosis or definite diagnosis. All of the patients agreed to donate the bio-specimen for scientific research use and signed the informed consent after admission. This study was approved by the ethical committee of Peking Union Medical College Hospital. 


\section{Statistics}

The IBM SPSS Statistics software 22.0 version and the GraphPad Prism software 5.0 version were applied for statistical analysis and for drawing the graphs. The differences of clinical-pathological variables were assessed using Wilcoxon signed-rank test, student $t$ test, ANOVA ( or Tukey's Multiple Comparison Test), chi-square test or Fisher's exact test when adequate. The Spearman correlation between CA19-9 and TBIL was analyzed. The receiver operating characteristic (ROC) curve was applied to compare the power of the increase-folds of CA19-9, TBIL and the ratio for differential diagnosis of MOJ and BOJ. The optimal cutoff value of CA19-9, TBIL and the ratio for differential diagnosis was determined by the Youden's index. A $P$ value less than 0.05 was considered to be statistically significant.

\section{Results}

\section{The basic information of the patients}

342 cases of biliary and pancreatic adenocarcinoma were enrolled. 182 cases were male and 160 cases were female. The median age was 63 years, ranging from 28 to 92 years. 166 cases were biliary and pancreatic benign diseases. 122 cases were male and 44 cases were female. The median age was 56 years, ranging from 13 to 98 years. The gender and the age of malignancies and benign diseases were significantly different $(P<0.0001$, Chi-square test test; $P<0.0001$, Wilcoxon signed-rank test). The IRSD was predominant in elderly male patients, which was similar to the previous reports[1]. The detail information of the etiologies of the MOJ and BOJ was listed in Table 1.

Table 1. The basic information of the patients

\begin{tabular}{|c|c|c|c|}
\hline Diagnosis & $\mathrm{N}$ & $\begin{array}{l}\text { Age } \\
\text { (Median,25\%IQR 75\%IQR) }\end{array}$ & $\begin{array}{l}\text { Gender } \\
\text { Male:Female(ratio) }\end{array}$ \\
\hline $\begin{array}{l}\text { Pancreatic ductal } \\
\text { adenocarcinoma }\end{array}$ & 138 & $62(54 \sim 69)$ & $71: 67(1.06)$ \\
\hline Cholangiocarcinoma & 118 & $63(57 \sim 69)$ & 64:54(1.19) \\
\hline $\begin{array}{l}\text { Periampullar } \\
\text { adenocarcinoma }\end{array}$ & 66 & $63(54 \sim 73)$ & $37: 29(1.28)$ \\
\hline $\begin{array}{l}\text { Gallbladder } \\
\text { adenocarcinoma }\end{array}$ & 17 & $61(52 \sim 67)$ & $7: 10(0.7)$ \\
\hline Cystadenocarcinoma & 3 & $57(51 \sim 68)$ & $1: 2(0.5)$ \\
\hline $\begin{array}{l}\text { IgG4 related sclerosing } \\
\text { cholangitis }\end{array}$ & 76 & $58(51 \sim 66.5)$ & $63: 13(4.85)$ \\
\hline Cholelithiasis & 48 & $58(43 \sim 71)$ & 31:17 (1.82) \\
\hline Inflammatory stricture & 14 & $44.5(32.5 \sim 58)$ & 9:5 (1.8) \\
\hline $\begin{array}{l}\text { Primary sclerosing } \\
\text { cholangitis }\end{array}$ & 13 & $38(27 \sim 43)$ & $7: 5(1.4)$ \\
\hline Adenoma & 11 & $55.5(40 \sim 61)$ & $6: 5(1.2)$ \\
\hline Biliary cyst & 4 & $39(35 \sim 45)$ & $2: 2(1.0)$ \\
\hline
\end{tabular}

\section{The differences of TBIL, CA19-9 and the ratio of $\mathrm{MOJ}$ and $\mathrm{BO}$ J}

\subsection{The increase-folds of TBIL of MOJ and $\mathrm{BOJ}$}

Among the 342 cases of the MOJ, the elevation of serum TBIL was less than 5 folds in 137 cases (40.1\%), 5 10 folds in 129 cases (37.7\%), more than 10 folds in 76 cases $(22.2 \%)$. Among the 166 cases of the BOJ, the elevation of serum TBIL was less than 5 folds in 89 cases $(53.6 \%), 5 \sim 10$ folds in 52 cases $(31.3 \%)$, more than 10 folds in 38 cases $(22.9 \%)$. The serum increase-folds of TBIL of MOJ was higher than that of BOJ $(P=0.0085$, Student $t$ test $)$ (Figure 1, supplementary material Table S1). The elevation of TBIL of cholangiocarcinoma was stronger than that of periampullar adenocarcinoma $(P=0.0163$, Tukey's Multiple Comparison Test) (Figure 2, supplementary material Table S2). The elevation of TBIL of PSC was significantly higher than that of cholelithiasis, IRSD and PSC ( $P<0.0001$, Tukey's Multiple Comparison Test) (Figure 2, supplementary material Table S3).

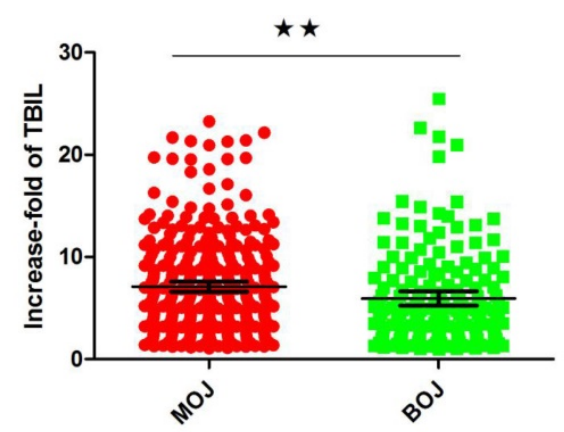

\section{A}

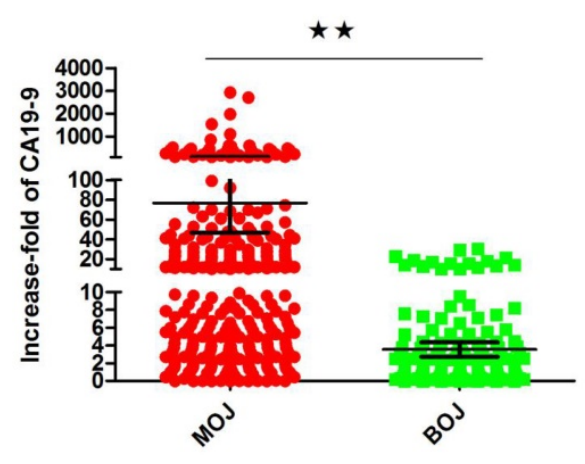

B

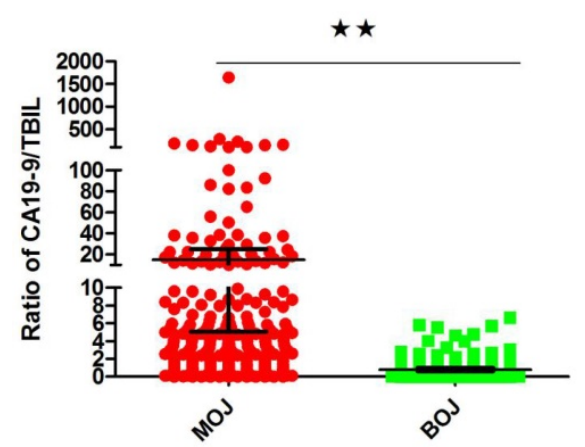


Figure 1. The differences of the increase-folds of TBIL, the increase-folds of CA19-9 and the ratio of increase-folds of CA19-9 to increase-folds of TBIL of MOJ and BOJ (median value \pm interquartile range): the elevations of serum TBIL, CA9-9 and the ratio of $\mathrm{MOJ}$ were significantly higher than those of $\mathrm{BO}$ J. **, $P<0.01$

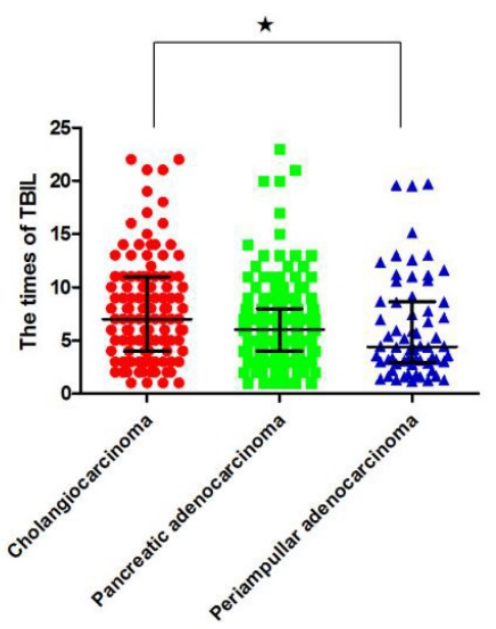

A

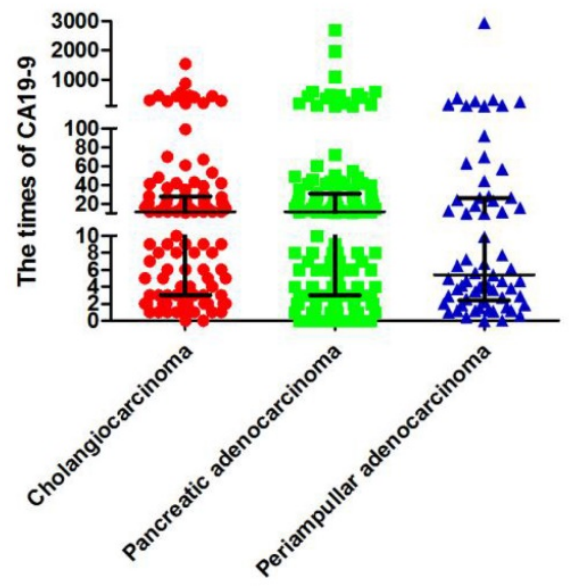

B

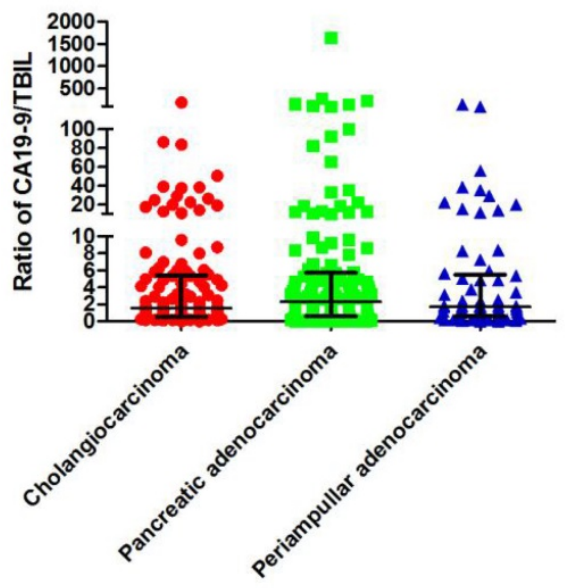

C

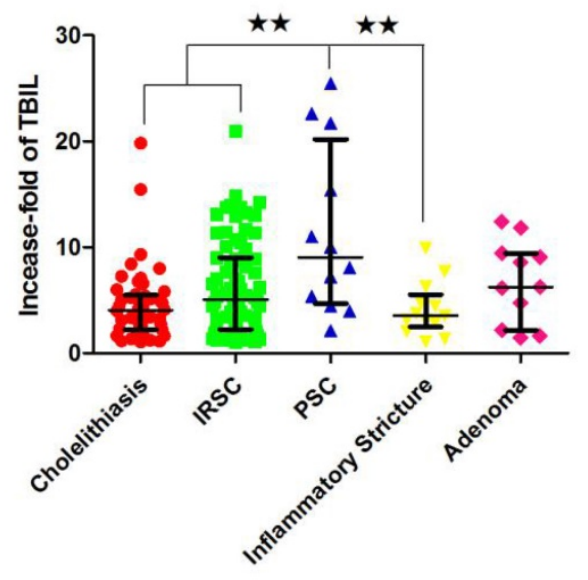

D

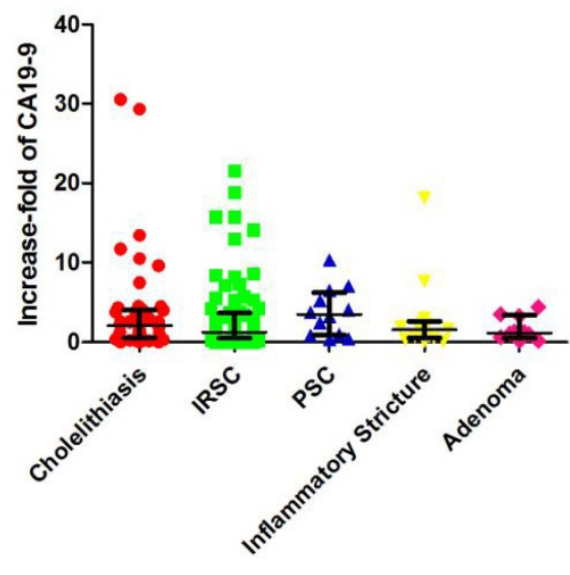

$\mathbf{E}$

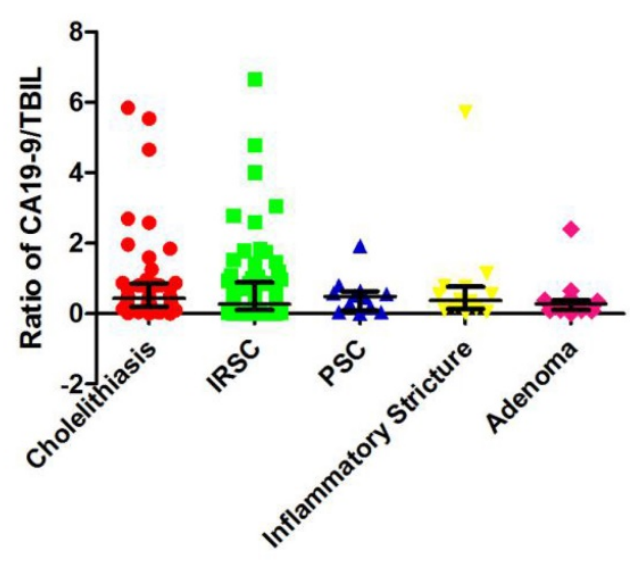

$\mathbf{F}$ 
Figure 2. The differences of the increase-folds of TBIL, the increase-folds of CA19-9 and the ratio of increase-folds of CA19-9 to increase-folds of TBIL of different etiologies of $\mathrm{MOJ}$ and $\mathrm{BOJ}$ (median value \pm interquartile range): In MOJ, the elevation of TBIL of cholangiocarcinoma was significantly higher than that of periampullary adenocarcinooma; In BOJ, the elevation of TBIL in PSC was significantly higher than that of cholelethiasis, IRSC and inflammatory stricture. $P<0.05 ; * *, P<0.01$ (Tukey's Multiple Comparison Test)

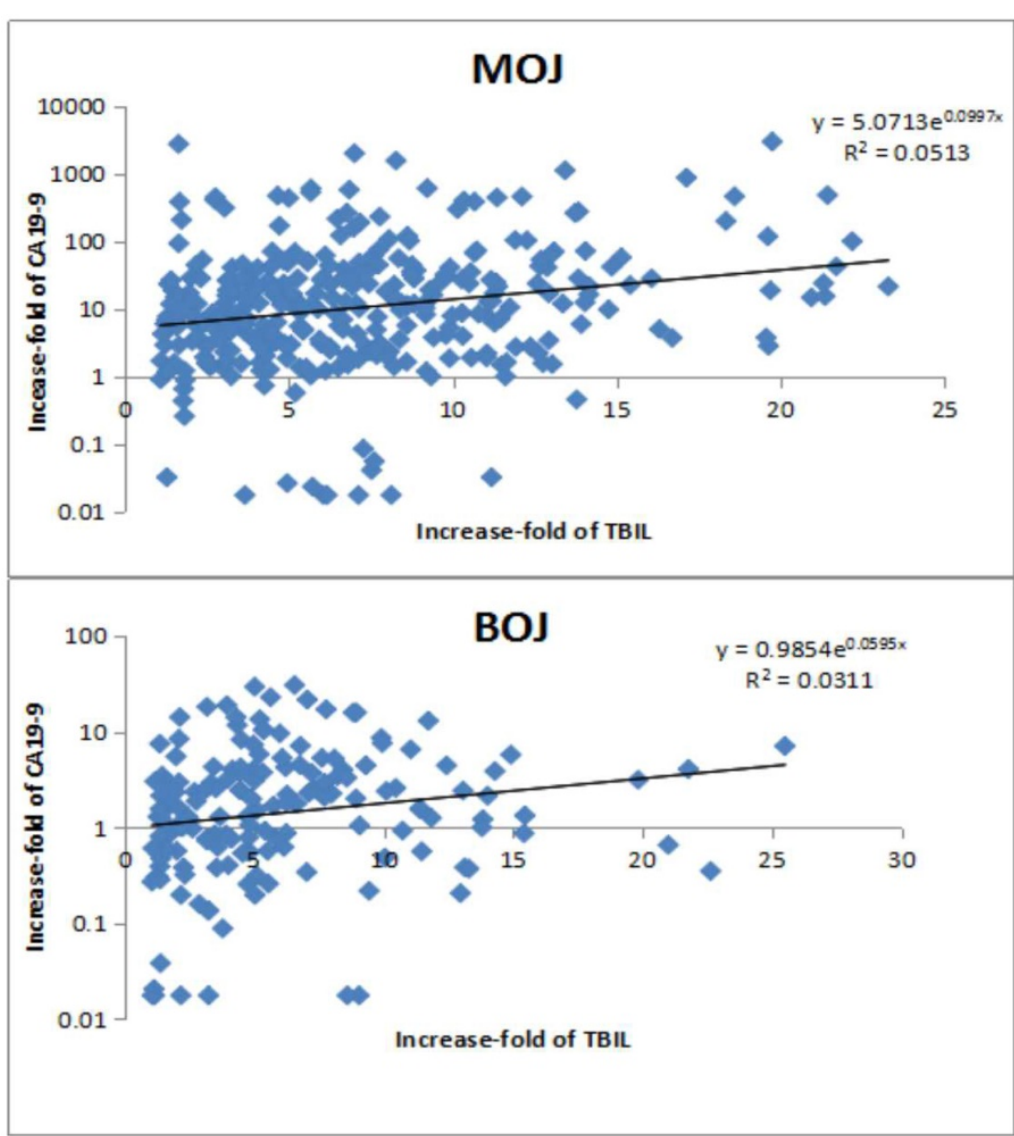

Figure 3. The correlations between increase-folds of CA199 and increase-folds of TBIL of MOJ and BOJ: In both BOJ and MOJ, the elevation of CA19-9 was positively correlated with the elevation of TBIL in an exponential model.

\subsection{The increase-folds of CA19-9 of $\mathrm{MOJ}$ and $\mathrm{BO}$}

Among the 342 cases of MOJ, the serum CA19-9 was normal in only 20 cases $(5.85 \%)$, the elevation was less than 5 folds in 100 cases $(29.2 \%), 5 \sim 10$ folds in 48 cases $(14.04 \%), 10 \sim 20$ folds in 55 cases $(16.08 \%)$, more than 20 folds in 119 cases (34.83\%). Among the 166 cases of BOJ, the serum CA19-9 was normal in 64 cases $(39.51 \%)$, the elevation was less than 5 folds in 70 cases $(49.30 \%), 5 \sim 10$ folds in 16 cases $(11.27 \%)$, 10 20 folds in 12 cases (8.45\%), more than 20 folds in 4 cases $(2.82 \%)$. The increase-fold of serum CA19-9 of MOJ was higher than that of BOJ $(P<0.0001$, Student $t$ test) (Figure 1, supplementary material Table $S 1$ ). The elevations of CA19-9 of the different etiologies of MOJ were not significantly different $(P>0.05$, ANOVA) (Figure 2, supplementary material Table S2). The elevations of CA19-9 of different etiologies of BOJ were not significantly different as well $(P>0.05$, Student $\mathrm{t}$ test) (Figure 2, supplementary material Table S3).

\subsection{The ratio of increase-folds of CA19-9 to increase-foldS of TBIL of MOJ and BOJ}

Among the 342 cases of MOJ, the ratio was less than 1.00 in 124 cases $(36.25 \%), 1 \sim 5$ in 125 cases (36.54\%), 5 10 in 42 cases (12.28\%), more than 10 in 51 cases $(14.93 \%)$. Among the 166 cases of BOJ, the ratio was less than 1.00 in 133 cases, 1 5 in 29 cases, 5 10 in 5 cases, more than 10 in 0 . The ratio of MOJ was significanlty higher than that of BOJ $(P=0.0053$, Student $t$ test) (Figure 1). The elevations of ratio of pancreatic adenocarcinoma, cholangiocarcinoma and periampullar adenocarcinoma were not significantly different (ANOVA, $P>0.05$ ) (Figure 2, supplemenetary material Table S2). The elevations of ratio in IRSC and cholelithiasis were not significantly different as well (ANOVA, $P>0.05$ ). (Figure 2, supplementary material Table S3)

2.4 The correlation between the increase-folds of TBIL and the increase-folds of CA19-9 of MOJ and $\mathrm{BO}$

In MOJ, the serum CA19-9 was positively 
correlated with the TBIL $\left(y=5.0713 \mathrm{e}^{0.0997 x}, \mathrm{R}^{2}=0.0513\right.$, Spearman's rank correlation coefficient, $P<0.0001)$. As well, in BOJ, the CA19-9 was positively related with the TBIL $\left(y=5.0713 \mathrm{e}^{0.0997 x}, \mathrm{R}^{2}=0.0513\right.$, Spearman's rank correlation coefficient, $P<0.0001)$. Therefore, these results showed the serum CA19-9 could be affected by the TBIL in both MOJ and BOJ (Figure 3).

\section{The differential diagnostic roles of the increase-folds of CA19-9, TBIL and the ratio for MOJ and BOJ}

The ROC curve showed that the area under curve (AUC) of the increase-folds of BIL, the increase-folds of CA19-9 and the ratio was 0.590, 0.815 and 0.889 for differential diagnosis of MOJ, respectively (Figure 4). According to the ROC curve, the Youden's index was calculated by the formula " sensitivity+ specificity-1". The maximum Youden's index represented the optimal cutoff value. The optimal value of the increase-folds of CA19-9 and the ratio was 4.5 (sensitivity $67.8 \%$, specificity $80.7 \%$ ) and 0.88 (sensitivity $69.96 \%$, specificity $82.71 \%$ ) for differential diagnosis of MOJ, respectively. When the cutoff value of the increase-folds of CA19-9 was determined to be 4.5 , the positive likelihood ratio LR $(+)$ was 3.51 , the negative likelihood ratio LR(-) was 0.40 , the accuracy was $74.0 \%$, the positive predictive value (PPV) was $91.3 \%$ and the negative predictive value (NPV) was $56.69 \%$. When the cutoff value of the ratio was determined to be 0.88 , the LR $(+)$ was 3.60, the LR (-) was 0.385 , the accuracy was $77.47 \%$, the PPV was $93.09 \%$ and the NPV was $57.30 \%$. The combination of the increase-folds of CA19-9 $(\geq 4.5)$ and the ratio $(\geq 0.88)$ further improved the specificity, accuracy up to $93.2 \%$ and $80.5 \%$, respectively. When the increase-folds of CA19-9 was more than 4.5 and the ratio was larger than 6.66 , the possibility of $\mathrm{BOJ}$ was excluded. After stratified analysis of the different etiologies of BOJ, the differential diagnostic power of the ratio was stronger than the increase-folds of CA19-9 or TBIL alone for cholelithiasis, IRSC and inflammatory stricture which indicated that the ratio had better potential to differentiate cholelithiasis, IRSC and inflammatory stricture of bile duct from MOJ, respectively (Figure 4). 

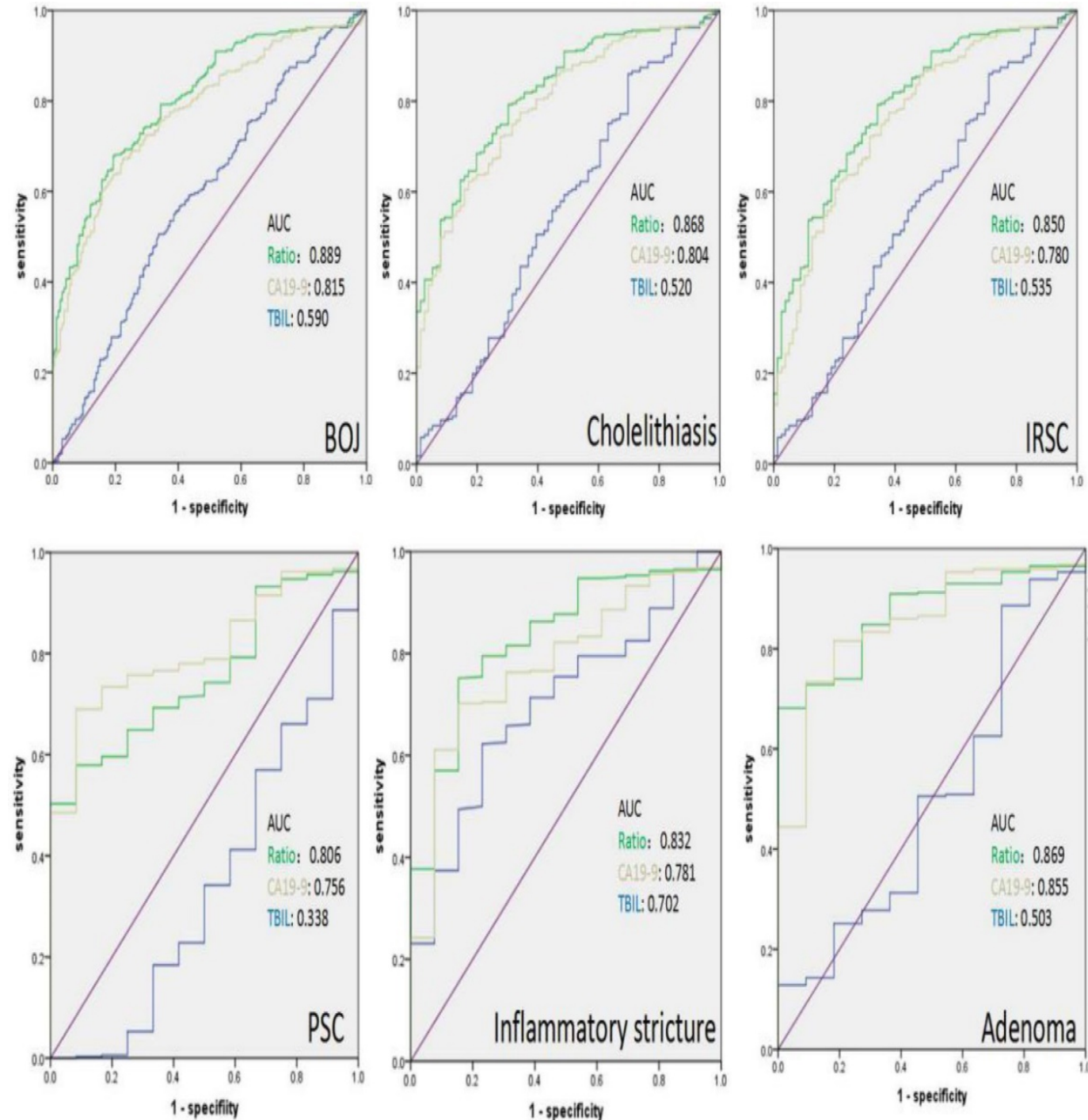

Figure 4. The AUC of the ROC of the different etiologies of BOJ: the AUC of the ratio was higher than that of the elevation of CA19-9 or TBIL alone in cholelithiasis, IRSC and inflammatory stricture of bile duct.

\section{Discussion}

For the patients with obstructive jaundice, accurate differential diagnosis is the most important step before further treatments. The serum CA19-9 is a widely used marker to help the differential diagnosis of the MOJ and BOJ[6]. The CA19-9 has been reported to be elevated in $70 \% \sim 90 \%$ pancreatic-biliary adenocarcinoma. However, the differential diagnostic power of CA19-9 can be disturbed by the hyperbilirubinemia and secondary inflammations[13]. How to improve the power of CA19-9 to discriminate between $\mathrm{MOJ}$ and $\mathrm{BOJ}$ was a classical topic with a long history, many strategies have been recommended, including a higher cutoff value, combination of other inflammatory markers, combination of the changes of CA19-9 after post-bile drainage et al.[3, 5, 14-17].However, these previous studies have some obvious limitations, including a small sample size ( none of these studies enrolled more than 300 patients. Although Kim et al.[17] announced that 981 patients were enrolled, only 114 patients were further analyzed.); the information of the spectrum of etiologies of MOJ and BOJ was not complete or totally missed in the previous studies; none of the previous reported enrolled IRSC; none of the previous studies validated the roles of the ratio of the elevation of CA19-9 to the elevation of TBIL for differential diagnosis of $\mathrm{MOJ}$ and $\mathrm{BOJ}$.

The IRSC is a newly defined rare autoimmune disease of bile duct characterized by high serum IgG4, stricture of bile duct and obstructive jaundice. It can be very difficult to distinguish IRSD from MOJ, especially when the serum IgG4 is normal. Up to now, there has never been a report about the direct comparison of the TBIL, CA19-9 and the ratio of IRSC with the other etiologies of BOJ and MOJ $[1,18]$. In 2014, a Japanese national survey reported 45 cases of IRSC [19]. Herein, we enrolled 76 cases of IRSC which could be the largest single center report. 
In this study, 508 cases of MOJ and MOJ with complete spectrum of etiologies were enrolled. The serum CA19-9 was elevated in $94.15 \%$ in MOJ, compared to $67.10 \%$ in BOJ. To improve the differential diagnostic power of CA19-9,some different higher cutoff value was recommended, such as $90 \mathrm{U} / \mathrm{ml}$, or $100 \mathrm{U} / \mathrm{ml}$, compared to the normal cutoff value, these higher cutoff values improved the specificity of MOJ [20]. In this study, by using a much larger number of patients than the previous reports, we found the serum of CA19-9 in most of the MOJ $(65.31 \%)$ was higher than 5 folds of the normal upper threshold value, to the contrary, only $22.54 \%$ of BOJ had a serum CA19-9 higher than 5 folds of the normal upper threshold value. The Youden's indexes from ROC curve determined the optimal cutoff value of the increasing fold of CA19-9 was $4.5(153 \mathrm{U} / \mathrm{ml})$. According to this cutoff value, the sensitivity was $67.80 \%$ and the specificity was $80.70 \%$ which were similar to the previous reports[15]. Therefore, a higher cutoff alone is not an ideal strategy to improve the differential diagnostic power of CA19-9.

Although the levels of TBIL in MOJ and BOJ were different, its differential diagnostic power was minimal (AUC $=0.590$ ). La Greca et al [3] reported that the serum CA19-9 was not associated with serum TBIL in neither MOJ nor BOJ, however in this study, we found that the serum CA19-9 was significantly associated with the serum TBIL in an exponential model in both MOJ and BOJ. It should be mentioned that there were only 102 cases enrolled in that study, in this study, the number of patients were much larger which made our results more reliable. Later, we tested the differential diagnostic power of the ratio of CA19-9 to TBIL. The ROC curve determined that the optimal cutoff value of the ratio was 0.88 . The ratio showed stronger power to differentiate BOJ from MOJ than the CA19-9 alone with a sensitivity of $69.96 \%$, a specificity of $82.71 \%$ and an accuracy of $77.47 \%$. Further, the combination of the ratio and the CA19-9 significantly improved the specificity from $80.7 \%$ up to $93.2 \%$, and only led to a slight decrease of the sensitivity from $67.8 \%$ to $62.3 \%$, and finally improved the accuracy from $74.0 \%$ to $80.5 \%$. In these 166 cases of BOJ, the combination of over 4.5 increase-folds of CA19-9 and 6.66 of the ratio, the possibility of BOJ could be excluded. La Greca et al.[3] also reported the adjustment of CA19-9 by TBIL and CRP to differentiate the MOJ from the BOJ in 2012. However, our study was significantly different from their study in many aspects. Firstly, in their study, only 102 patients were enrolled which was much smaller than our study; Secondly, although they adjusted the CA19-9 by division of TBIL, they did not define the optimal cutoff value of the ratio and the differential roles of combination of the ratio and the elevation of CA19-9 was not tested in their study; The last, and also the most important point is that our results showed that the best way to improve the power of CA19-9 was the combination of the ratio $(\geq 0.88)$ and the increase-folds of CA19-9 $(\geq 4.5)$ which improved the specificity, accuracy up to $93.2 \%$ and $80.5 \%$ and these results was much better than the ratio of CA19-9 to CRP which was recommended by their study.

In the workshop of clinical practice, it could be relatively easier to diagnose cholelithiasis[21, 22], however sometimes, it could be very difficult to differentiate IRSC[23], inflammatory stricture of bile duct $[24,25]$ from malignant tumors. In this study, we found that IRSD was predominant in elderly male patients, which was in accordance with the previous reports. The results showed that the elevation of CA19-9 of IRSD was not significantly different the other etiologies of BOJ. After stratified analysis, the ratio showed stronger power than the increase-folds of CA19-9 alone to differentiate IRSD and inflammatory stricture of bile duct from MOJ.

In conclusion, the highlight points of this study included: (1) According to the rigid criteria, 508 cases of obstruction jaundice patients were enrolled which should be the largest report and also the one with most complete spectrum of etiologies of BOJ and $\mathrm{MOJ}$, compared to the precious similar reports; (2) This study enrolled 76 of IRSD, which should be the largest single center report, and for the first time, the TBIL, CA19-9 and the ratio of IRSD were directly compared with the other etiologies of $\mathrm{BOJ}$ and MOJ; (3) By using this large number of patients, we found the optimal cutoff value of increase-folds of CA19-9(4.5) and the ratio(0.88) to differentiate BOJ from MOJ; (4) For the first time, we demonstrated the assumption that the combination of the increase-folds of CA19-9 and the ratio of CA19-9 to TBIL was superior to the increase-folds of CA19-9 alone to discriminate between $\mathrm{MOJ}$ and $\mathrm{BOJ}$.

\section{Abbreviations}

AUC: area under curve; BOJ: benign obstructive jaundice; CA19-9: carbohydrate antigen 19-9; MOJ: malignant obstructive jaundice; IRSC: IgG4 related sclerosing cholangitis; ROC curve: receiver operating characteristic curve; PSC: primary sclerosing cholangitis; TBIL: total bilirubin.

\section{Supplementary Material}

Supplementary figures and tables. http://www.jcancer.org/v09p1804s1.pdf

\section{Acknowledgements}

The authors sincerely express their thanks to the 
support and assistance from the colleagues of department of general surgery of Peking Union Medical College Hospital.

\section{Ethics approval and consent to participate}

The study was approved by the ethical committee of Peking Union Medical College Hospital. Informed written consent was obtained from all patients and is available on request.

\section{Consent to publish}

All participants signed informed consent including consent for publication.

\section{Availability of data and materials}

The datasets used and analyzed in this study are available from the corresponding author on reasonable request.

\section{Funding}

This work was supported by National Natural Science Foundation of China (81502068, 81673023 and 81272573) and Beijing Natural Science Foundation of China (7172177).

\section{Authors' Contributions}

$\mathrm{XDH}, \mathrm{WL}$ and QFL designed the experiments. WL, QFL, WQW, PHW, JMC, TH, NZ, BLL and QQ collected and analyzed the data. QFL wrote the manuscript. WL revised the manuscript.

\section{Trial registration}

ClinicalTrials.gov Identifier -NCT02654288 (Retrospectively Registered 26/1/2016).

\section{Competing Interests}

The authors have declared that no competing interest exists.

\section{References}

1. Tanaka A, Tazuma S, Okazaki K, Nakazawa T, Inui K, Chiba T, et al. Clinical Features, Response to Treatment, and Outcomes of IgG4-Related Sclerosing Cholangitis. Clin Gastroenterol Hepatol. 2017; 15: 920-6.

2. Ray K. Pancreatic cancer: Biomarkers for the early detection of PDAC. Nat Rev Gastroenterol Hepatol. 2017; 14: 504-5.

3. La Greca G, Sofia M, Lombardo R, Latteri S, Ricotta A, Puleo S, et al. Adjusting CA19-9 values to predict malignancy in obstructive jaundice: influence of bilirubin and C-reactive protein. World J Gastroenterol. 2012; 18: 4150-5.

4. Hogendorf P, Skulimowski A, Durczynski A, Kumor A, Poznanska G, Olesna A, et al. A Panel of CA19-9, Ca125, and Ca15-3 as the Enhanced Test for the Differential Diagnosis of the Pancreatic Lesion. Dis Markers. 2017; 2017: 8629712.

5. Marrelli D, Caruso S, Pedrazzani C, Neri A, Fernandes E, Marini M, et al CA19-9 serum levels in obstructive jaundice: clinical value in benign and malignant conditions. Am J Surg. 2009; 198: 333-9.

6. Su SB, Qin SY, Chen W, Luo W, Jiang HX. Carbohydrate antigen 19-9 for differential diagnosis of pancreatic carcinoma and chronic pancreatitis. World J Gastroenterol. 2015; 21: 4323-33.

7. Kaur S, Smith LM, Patel A, Menning M, Watley DC, Malik SS, et al. A Combination of MUC5AC and CA19-9 Improves the Diagnosis of Pancreatic Cancer: A Multicenter Study. Am J Gastroenterol. 2017; 112: 172-83.

8. Xiao J, Li G, Yang G, Jia C, Li B. Case report: A female case of isolated IgG4-related sclerosing cholangitis mimicking cholangiocarcinoma. Medicine (Baltimore). 2017; 96: e6542
9. Yata M, Suzuki K, Furuhashi N, Kawakami K, Kawai Y, Naganawa S. Comparison of the multidetector-row computed tomography findings of IgG4-related sclerosing cholangitis and extrahepatic cholangiocarcinoma. Clin Radiol. 2016; 71: 203-10.

10. Chapman R, Fevery J, Kalloo A, Nagorney DM, Boberg KM, Shneider B, et al. Diagnosis and management of primary sclerosing cholangitis. Hepatology. 2010; 51: 660-78.

11. Consensus on the diagnosis and management of primary sclerosing cholangitis (2015 version). Zhonghua Gan Zang Bing Za Zhi. 2016; 24: 14-22.

12. Ohara H, Okazaki K, Tsubouchi H, Inui K, Kawa S, Kamisawa T, et al. Clinical diagnostic criteria of IgG4-related sclerosing cholangitis 2012. J Hepatobiliary Pancreat Sci. 2012; 19: 536-42.

13. Sheen-Chen SM, Sun CK, Liu YW, Eng HL, Ko SF, Kuo CH. Extremely elevated CA19-9 in acute cholangitis. Dig Dis Sci. 2007; 52: 3140-2.

14. Duraker N, Hot S, Polat Y, Hobek A, Gencler N, Urhan N. CEA, CA 19-9, and CA 125 in the differential diagnosis of benign and malignant pancreatic diseases with or without jaundice. J Surg Oncol. 2007; 95: 142-7.

15. Morris-Stiff G, Teli M, Jardine N, Puntis MC. CA19-9 antigen levels can distinguish between benign and malignant pancreaticobiliary disease. Hepatobiliary Pancreat Dis Int. 2009; 8: 620-6.

16. Gibor U, Perry ZH, Netz U, Glazer Y, Laufer L, Kirshtein B. CA 19-9 in the presence of obstructive jaundice due to Mirizzi syndrome. Isr Med Assoc J. 2015; 17: 60-1.

17. Kim MS, Jeon TJ, Park JY, Choi J, Shin WC, Park SE, et al. Clinical Interpretation of Elevated CA 19-9 Levels in Obstructive Jaundice Following Benign and Malignant Pancreatobiliary Disease. Korean J Gastroenterol. 2017; 70: $96-102$.

18. Moon SH, Kim MH, Lee JK, Baek S, Woo YS, Cho DH, et al. Development of a scoring system for differentiating IgG4-related sclerosing cholangitis from primary sclerosing cholangitis. J Gastroenterol. 2017; 52: 483-93.

19. Tanaka A, Tazuma $\mathrm{S}$, Okazaki $\mathrm{K}$, Tsubouchi $\mathrm{H}$, Inui $\mathrm{K}$, Takikawa $\mathrm{H}$ Nationwide survey for primary sclerosing cholangitis and IgG4-related sclerosing cholangitis in Japan. J Hepatobiliary Pancreat Sci. 2014; 21: 43-50.

20. Garcea G, Ngu W, Neal CP, Dennison AR, Berry DP. Bilirubin levels predict malignancy in patients with obstructive jaundice. HPB (Oxford). 2011; 13: 426-30.

21. Neitlich T, Neitlich J. The imaging evaluation of cholelithiasis in the obese patient-ultrasound vs CT cholecystography: our experience with the bariatric surgery population. Obes Surg. 2009; 19: 207-10.

22. de Bari O, Wang TY, Liu M, Paik CN, Portincasa P, Wang DQ. Cholesterol cholelithiasis in pregnant women: pathogenesis, prevention and treatment. Ann Hepatol. 2014; 13: 728-45.

23. Maeda M, Shimada K. A case of IgG4-related sclerosing cholangitis mimicking an intrahepatic cholangiocellular carcinoma. Jpn J Clin Oncol. 2012; 42: 153.

24. Katabathina VS, Dasyam AK, Dasyam N, Hosseinzadeh K. Adult bile duct strictures: role of MR imaging and MR cholangiopancreatography in characterization. Radiographics. 2014; 34: 565-86.

25. Chathadi KV, Khashab MA, Acosta RD, Chandrasekhara V, Eloubeidi MA, Faulx AL, et al. The role of endoscopy in ampullary and duodenal adenomas. Gastrointest Endosc. 2015; 82: 773-81. 